\title{
Planejamento agregado na indústria de nutrição animal sob incertezas
}

\author{
Diego Barreiros Augusto ${ }^{\mathrm{a}}$, Douglas Alema*, Eli Angela Vitor Toso ${ }^{\mathrm{a}}$ \\ a*Universidade Federal de São Carlos, Sorocaba, SP, Brasil, dougepai@yahoo.com.br
}

\begin{abstract}
Resumo
Um dos desafios para o planejamento da produção na indústria de nutrição animal consiste em determinar quanto produzir de cada produto em cada período, considerando que existem incertezas associadas às operações de setup, que os produtos são perecíveis e que a capacidade produtiva deve ser ajustada num ambiente de demanda estocástica caracterizada pela sazonalidade dos produtos e das matérias-primas. Este trabalho investiga um problema de planejamento agregado da produção em uma planta que produz suplementos para nutrição animal. Para lidar com esse problema, propôs-se uma extensão do problema clássico de dimensionamento de lotes com restrição de capacidade para incorporar decisões sobre vendas perdidas (lost sales) e as incertezas inerentes ao planejamento da produção: demandas, tempos de preparação e taxa de perecibilidade dos produtos. Para gerar soluções menos sensiveis às variações dos cenários, desenvolveu-se um modelo estocástico com aversão ao risco baseado numa medida de risco do tipo semidesvio absoluto. Analisando-se o valor esperado da informação perfeita e o valor da solução estocástica, confirmou-se o desempenho superior do modelo de programação estocástica no tratamento das incertezas. Além disso, os resultados indicaram que é possivel reduzir significativamente a variabilidade dos custos de segundo estágio sem sacrificar demasiadamente o custo total esperado.
\end{abstract}

Palavras-chave

Problema de dimensionamento de lotes capacitado com lost sales e perecibilidade. Programação estocástica de dois estágios. Gestão de risco. Indústria de nutrição animal.

\section{Introdução}

A indústria de produtos de nutrição animal é um importante elo na cadeia agroindustrial da produção pecuária brasileira, uma vez que esse setor é responsável pela produção de rações comerciais, pré-misturas, complexos vitamínicos e outros produtos para alimentação de diversas espécies animais. Atualmente, a produção dessa indústria representa $6,5 \%$ do PIB brasileiro, gera milhares de empregos diretos e indiretos e é responsável por 18\% das exportações do agronegócio nacional (Sindicato Nacional da Indústria de Alimentação Animal, 2013). Na última década, a produção nacional nesse segmento vem crescendo significativamente. No entanto, esse crescimento é bastante sensível às oscilações econômicas no mercado. Entre 2009 e 2010, por exemplo, a produção aumentou 5,3\%, com 61,4 milhões de toneladas de rações produzidas no ano e uma movimentação de $\mathrm{R} \$ 33$ bilhões apenas com matérias-primas. Já em
2012, embora as projeções iniciais indicassem a expectativa de um crescimento de $2,8 \%$, houve uma retração de 2,3\% em relação à quantidade de ração produzida e de $17 \%$ em relação à quantidade de suplementos produzidos em 2011 (Sindicato Nacional da Indústria de Alimentação Animal, 2013).

Assim como outros setores industriais, o setor de nutrição animal busca aumentar sua competitividade no cenário mundial, incorporando rapidamente novas informações de mercado e tecnologias para melhorar o desempenho das suas decisões no curto e médio prazo, principalmente. Como consequência da maior complexidade de produtos e sistemas de produção e dos altos custos envolvidos, é relevante a busca por novas ferramentas para apoiar as decisões relacionadas ao planejamento e programação da produção. 0 planejamento e a programação da produção nessa indústria apresentam dificuldades relacionadas à 
melhor utilização dos recursos produtivos, redução de perdas com tempos de preparação, períodos de ociosidade das linhas, entre outros.

Há questões específicas do setor de nutrição animal que normalmente não são contempladas por modelos clássicos de dimensionamento de lotes propostos na literatura (Drexl \& Kimms, 1997; Karimi et al., 2003; Pochet \& Wolsey, 2006; Brahimi et al., 2006; Jans \& Degraeve, 2008), como a sazonalidade dos produtos e as incertezas relacionadas à demanda e ao processo de produção. Segundo Toso et al. (2008), como os meses de junho a agosto correspondem à época de seca, o consumo de rações minerais para bovinos aumenta e, como os pedidos dos clientes podem surgir aleatoriamente ao longo do horizonte de planejamento, a variação da demanda ao longo dos períodos é grande. Assim, as empresas precisam buscar alternativas para equilibrar demanda e capacidade de produção, como a construção de estoques de segurança nos períodos de capacidade mais folgada, atraso na entrega de pedidos ou mesmo a não produção de parte dos produtos (lost sales), utilização de horas extras ou subcontratação, adequação dos níveis de força de trabalho ao longo dos períodos, entre outras.

Outro desafio para o planejamento da produção na indústria de nutrição animal refere-se às operações de preparação (setups). A execução dessas atividades depende do estado de funcionamento do maquinário e do trabalho dos operadores que realizam a limpeza dos misturadores manualmente e, portanto, ela está sujeita a falhas humanas e variabilidade. Embora os custos com a limpeza do misturador sejam baixos, considerar a variabilidade do tempo gasto nessa atividade é importante, pois o mesmo onera a capacidade produtiva, que costuma ser bastante limitada.

0 risco de perecibilidade associado às matérias-primas e aos produtos acabados é outra particularidade que dificulta o planejamento e controle da produção. As taxas de perecibilidade são difíceis de estimar, pois o produto consiste em uma mistura de diversos ingredientes com prazos de validade diferentes. Além disso, as condições de armazenagem influenciam a taxa de deterioração de alguns ingredientes. Por exemplo, alguns ingredientes são mais higroscópicos e podem ter sua deterioração acelerada em ambientes com maior umidade. Ao se considerar o horizonte de tempo para planejamento agregado é importante incorporar as taxas de perecibilidade no processo decisório, pois elas influenciam as decisões sobre níveis de estoque de antecipação, visto que o tempo de vida útil dos produtos na fábrica é de cerca de 90 dias. Diversos modelos de planejamento da produção incorporam decisões relacionadas à deterioração e perecibilidade de produtos. De acordo com Alfares et al. (2005), esses modelos podem ser classificados em relação ao tempo de vida útil (lifetime) como fixos ou aleatórios e, com relação à taxa de deterioração, como constantes ou variáveis. Alguns modelos consideram o tempo de vida útil e controlam a quantidade deteriorada no balanceamento de estoques (Pahl et al., 2011), enquanto outros trabalhos consideram uma taxa de perecibilidade associada aos produtos, e.g., Ahumada \& Villalobos (2008), Costa et al. (2014) e Amorim et al. (2013).

A produção em grandes volumes ajuda a reduzir o lead time no atendimento da demanda aleatória, ou devido a transtornos diversos, e ainda permite uma possível economia nos tempos de preparação. No entanto, a manutenção e o gerenciamento de grandes estoques de segurança das plantas ocultam fontes de desperdício (deterioração do estoque) e problemas de planejamento e controle da produção mal resolvidos, como contratações desnecessárias de funcionários e/ou turnos de trabalho adicionais. Assim, o planejamento da produção na indústria de suplementos para nutrição animal deve incorporar as incertezas inerentes ao processo de produção para permitir o compromisso entre diversas decisões conflitantes do dimensionamento de lotes.

Nesse contexto, o presente trabalho propõe uma extensão do clássico problema de dimensionamento de lotes com restrição de capacidade e lost sales para incorporar demandas, tempos de preparação e taxas de perecibilidade incertos. A incerteza dos três parâmetros é aproximada via um conjunto discreto e finito de realizações ou cenários, cada qual associado a uma probabilidade de ocorrência. 0 modelo matemático resultante considera, simultaneamente, todos os cenários, a fim de fornecer uma solução de compromisso entre as decisões de primeiro estágio "aqui e agora", tomadas antes do conhecimento das variáveis aleatórias, e as decisões de segundo estágio "espere e veja”, determinadas após a observação das variáveis aleatórias. Uma vez que reimplementar as decisões para cada cenário materializado pode ser operacionalmente custoso, o modelo de dois estágios é estendido para agregar um critério de aversão ao risco que minimiza a variabilidade dos custos de segundo estágio, de acordo com a propensão ao risco do tomador de decisão.

A ideia de incorporar as incertezas dos problemas de planejamento da produção data dos trabalhos pioneiros de Bellman (1957), Dantzig (1955), Charnes \& Cooper (1959) e Bellman \& Zadeh (1970). Desde então, acadêmicos e profissionais têm desenvolvido e aplicado diversas metodologias para tratar das incertezas em problemas práticos. 0 leitor é remetido para Sahinidis (2004) e Mula et al. (2006) para revisões sobre otimização sob incerteza e especificamente no contexto de planejamento de produção, respectivamente. 
Verderame et al. (2010) preconiza que a programação estocástica é uma das filosofias de modelagem mais bem-sucedidas para lidar com as incertezas no processo de planejamento e programação da produção, provavelmente porque a modelagem utilizando cenários pode ser bastante intuitiva e as técnicas de otimização linear/inteira podem ser estendidas para os modelos estocásticos sem grandes dificuldades. Uma alternativa à utilização da programação estocástica é uma abordagem aparentemente introduzida no trabalho de Mulvey et al. (1995), denominada pelos autores otimização robusta e hoje mais popularmente conhecida por programação estocástica com aversão ao risco. Tal metodologia agrega restrições ou medidas de risco ao problema de programação estocástica para gerar uma série de soluções progressivamente menos sensíveis aos diferentes cenários. Ao mesmo tempo, o problema com aversão ao risco é mais adequado para modelar situações em que o risco de cenários de pior caso deve ser evitado (Alem \& Morabito, 2013 ).

0 artigo está organizado da seguinte maneira: a seção 2 descreve o processo produtivo da indústria de suplementos para nutrição animal; a seção 3 desenvolve um modelo determinístico para representar o problema de planejamento agregado da produção dessa indústria, considerando as principais características do processo de produção; a seção 4 apresenta um modelo de programação estocástica de dois estágios com recurso para incorporar os parâmetros estocásticos ao problema; a seção 5 propõe um modelo de programação estocástica com aversão ao risco para controlar a variabilidade dos custos de segundo estágio; a seção 6 analisa os resultados computacionais dos modelos propostos; e, finalmente, a seção 7 discute as considerações finais e possíveis trabalhos futuros.

\section{Processo de produção de uma unidade de suplementos vitamínicos}

0 processo de produção na unidade de suplementos para nutrição animal é intermitente e acontece em bateladas, tendo como limitação o volume de produção dos misturadores de, aproximadamente, 2 mil litros. Como a quantidade máxima produzida em quilogramas varia de produto para produto, uma vez que as suas densidades são diferentes, é comum a unidade de produção ser considerada uma batelada. Por exemplo, uma batelada de núcleos/rações equivale a 2 toneladas, enquanto as bateladas de premixes e sais minerais equivalem a 1,44 e 2,4 toneladas, respectivamente (Toso et al., 2008).

Tipicamente, o processo produtivo na unidade de suplementos para nutrição animal envolve o conjunto de operações: (i) Dosagem de matérias-primas: os macroingredientes são pesados automaticamente em balanças, levando-se em conta a formulação do produto e o número de bateladas. Os microingredientes são pesados separadamente (utilizando-se balanças menores) e colocados no misturador manualmente. (ii) Pré-mistura: os materiais já pesados aguardam a pesagem do resto dos ingredientes para que a mistura seja realizada. (iii) Mistura dos ingredientes: com todos os ingredientes pesados, segue-se o processo de mistura, que ocorre em três etapas: mistura a seco, adição de líquidos e mistura úmida. (iv) Pós-misturador. a mistura pronta é descarregada no pós-misturador. ( $v$ Ensaque: do pós-misturador o produto é transportado para outro processo, no qual é realizado o ensaque para posterior expedição. Embora o processo seja constituído por várias etapas, ele pode ser considerado monoestágio, pois as etapas são realizadas de maneira sequencial. A Figura 1 ilustra a sequência mais comum em que essas operações são realizadas. Mais detalhes sobre o processo de produção na indústria de nutrição animal podem ser vistos em Toso et al. (2008).

\section{Um modelo determinístico de dimensionamento de lotes com lost sales e perecibilidade}

Este estudo propõe investigar o problema de planejamento da produção na indústria de ração animal considerando-se a perecibilidade dos produtos e a possibilidade de lost sales. Como o objetivo do problema é determinar um plano ótimo de produção para um horizonte de planejamento de médio prazo, as decisões operacionais foram agregadas e a tarefa de sequenciamento não foi considerada, diferentemente de Toso et al. (2009) e Clark et al. (2010).

Para modelar a perecibilidade dos produtos dentro de um contexto de planejamento agregado, foi incorporada ao modelo uma taxa de perecibilidade que pode ser alterada a cada período, por exemplo, produtos mais higroscópicos em períodos de maior umidade podem ter uma taxa de perecibilidade maior.

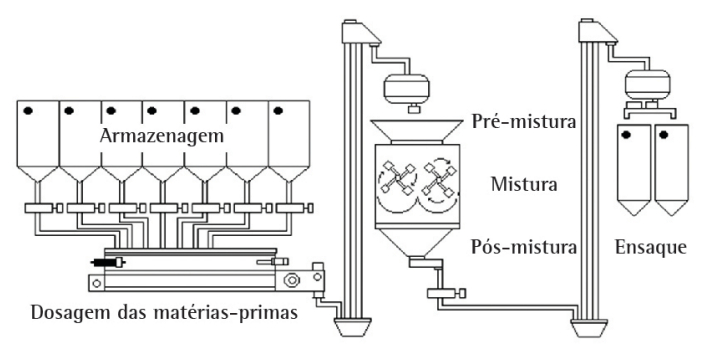

Figura 1. Etapas do processamento em uma planta de suplementos vitamínicos. 
A utilização dessa taxa, ao invés de controlar o tempo de vida útil, pode considerar um valor de perdas de acordo com o período de produção em questão e suas características. Esse critério de obsolescência é mais viável no planejamento agregado, visto que os produtos podem ser produzidos no início ou no final de um período e isso influencia o tempo de deterioração. Para descrever o modelo determinístico, considere a notação a seguir:

Parâmetros determinísticos:

- $s_{i t}$ - Custo de preparação do produto $i$ no período $t$;

- $o_{t}$ - Custo de hora extra no período $t$

- $h_{i t}^{+}$- Custo de estocagem do produto $i$ no período $t$;

- $h_{i t}^{-}$- Penalidade por não atender a demanda do produto $i$ no período $t$

- $C_{t}$ - Capacidade disponível no período $t$;

- $P_{i t}$ - Tempo de produção do produto $i$ no período $t$;

- $S_{i t}$ - Tempo de preparação do produto $i$ no período $t$;

- $D_{i t}$ - Demanda do produto $i$ no período $t$;

- $U_{t}$ - Disponibilidade máxima de hora extra no período $t$

- $R_{i t}$ - Taxa de perecibilidade do produto $i$ no período $t$. Variáveis de decisão:

- $Q_{i t}$ - Volume de produção do produto $i$ no período $t$;

- $l_{i t}^{+}$- Estoque do produto $i$ ao final do período $t$;

- $L_{i t}$ - Lost sales do produto $i$ ao final do período $t$;

- $Y_{i t}$ - Variável binária que indica se o produto $i$ é produzido no período $t\left(Y_{\mathrm{it}}=1\right)$ ou não $\left(Y_{\mathrm{it}}=0\right)$.

- $O_{t}$ - Hora extra utilizada no período $t$.

0 modelo matemático inteiro-misto de dimensionamento de lotes com lost sales e perecibilidade pode ser escrito como:

$$
\begin{array}{ll}
\min & \sum_{i \in I} \sum_{t \in T}\left(s_{i t} \cdot Y_{i t} h_{i t}^{+}+\cdot I_{i t}^{+}+h_{i t}^{-} \cdot L_{i t}\right)+\sum_{t \in T} o_{t} \cdot O_{t} \\
\text { s. a: } & \left\{\begin{array}{l}
Q_{i t}+\left(1-R_{i t}\right) \cdot I_{i(t-1)}^{+}+L_{i t}=D_{i t}+I_{i t}^{+}, \quad i \in I, t \in T \\
L_{i t} \leq D_{i t}, \quad i \in I, t \in T \\
Q_{i t} \leq\left(C_{t}+U_{t}\right) \cdot Y_{i t} \\
\sum_{i \in I}\left(P_{i t} \cdot Q_{i t}+S_{i t} \cdot Y_{i t}\right) \leq C_{t}+O_{t}, \quad t \in T \\
O_{t} \leq U_{t}, \quad t \in T \\
I_{i t}^{+}, L_{i t}, O_{t} \geq 0, \quad i \in I, t \in T \\
Q_{i t} \geq 0, Y_{i t} \in\{0,1\}, \quad i \in I, t \in T
\end{array}\right.
\end{array}
$$

A função objetivo do modelo de dimensionamento de lotes (1) consiste na minimização da soma dos custos de estocagem, preparação, lost sales e horas extras. A Equação (2) apresenta o balanceamento de estoques entre produção, entregas, estocagem e lost sales. Sem perda de generalidade, admite-se que os estoques iniciais são nulos. A proporção $R_{i t}$ indica a porcentagem do produto $i$ em estoque ao final do período $t-1$ que são considerados impróprios para comercialização como resultado do risco de perecibilidade. Note-se que se $R_{i t}$ é igual a 1 , toda a quantidade em estoque do período $t-1$ é perdida. Assim, não é possível atender a demanda do período $t$ a partir desse estoque. A restrição (3) define que o número de pedidos não atendidos não pode ser superior à demanda de cada produto em cada período. A restrição (4) garante que o tempo gasto com a produção e preparação em cada período não ultrapasse a capacidade de produção da fábrica, somada às horas extras trabalhadas naquele período. A restrição (5) impõe que um produto só será produzido caso o setup necessário à sua produção tenha sido realizado. 0 uso de horas extras é limitado ao máximo disponível por período, como ilustra a restrição (6). Os domínios das variáveis de decisão são mostrados nas restrições (7) e (8).

\section{Planejamento da produção em uma unidade de suplementos vitamínicos via programação estocástica}

Com base nas discussões anteriores, considerou-se que as demandas, taxas de perecibilidade e tempos de preparação são variáveis aleatórias que podem ser razoavelmente bem aproximadas por um conjunto discreto e finito de realizações ou cenários $W=\{1, \ldots, \omega, \ldots, \Xi\}$. A cada cenário é designado um peso $\pi$ que representa a sua chance de ocorrência, de tal forma que $\pi_{\omega}>0$ e $\Sigma_{\omega \in W} \pi_{\omega}=1$. Todos os cenários são considerados simultaneamente dentro de um problema de otimização de grande porte. No contexto da programação estocástica de dois estágios, as variáveis de decisões são divididas em dois conjuntos: antes e depois da ocorrência das variáveis aleatórias. As decisões anteriores à sua ocorrência são classificadas como decisões de primeiro estágio (here-and-now) e as decisões tomadas após as ocorrências das variáveis aleatórias são classificadas como decisões de segundo estágio (wait-and-see).

Neste trabalho, as quantidades produzidas $Q_{\mathrm{it}}$ são consideradas variáveis de primeiro estágio. Como a produção requer que o maquinário esteja preparado, a variável que controla a realização de setups $Y_{\mathrm{it}}$ também é de primeiro estágio. Com a realização das variáveis aleatórias, o modelo busca corrigir eventuais déficits associados à solução de primeiro estágio por meio de ações corretivas ou variáveis de segundo estágio, ou seja, estoques, horas extras e lost sales. Em outras palavras, como as decisões de produção e de setup já foram tomadas antes da realização das variáveis aleatórias, os custos totais de produção são minimizados com o ajuste dos níveis de estoque, a utilização das horas extras e a definição das lost sales. 
Portanto, temos os seguintes parâmetros estocásticos e variáveis de segundo estágio:

\section{Parâmetros estocásticos:}

- $D_{\text {ito }}$ - Demanda do item $i$ no período $t$ no cenário $\omega$;

- $R_{\text {ito }}$ - Proporção de perda por questões de perecibilidade do produto $i$ no período $t$ no cenário $\omega$;

- $S_{\text {ito }}$ - Tempo de preparação do produto $i$ no período $t$ no cenário $\omega$;

- $\pi_{\omega}$ - Probabilidade do cenário $\omega$ ocorrer. Variáveis de segundo estágio:

- $l_{\text {ito }}^{+}$- Estoque do item $i$ ao final do período $t$ no cenário $\omega$;

- $L_{\text {ito }}$ - Lost sales do item $i$ ao final do período $t$ no cenário $\omega$;

- $O_{\text {to }}$ - Hora extra utilizada no período $t$ no cenário $\omega$.

0 modelo matemático de programação estocástica de dois estágios com recurso pode ser escrito da seguinte forma:

$$
\min \sum_{i \in I \in T} \sum_{t \in T} s_{i t} \cdot Y_{i t}+\sum_{\omega \in W} \pi_{\omega} \cdot\left[\sum_{i \in I} \sum_{t \in T}\left(h_{i t}^{+} \cdot I_{i t \omega}^{+}+h_{i t}^{-} \cdot L_{i t \omega}\right)+\sum_{t \in T} o_{t} \cdot O_{t \omega}\right]
$$

$$
\text { s. a: }\left\{\begin{array}{c}
\text { Restrições determinísticas (4) e }(8) \\
Q_{i t}+\left(1-R_{i t \omega}\right) \cdot I_{i(t-1) \omega}^{+}+L_{i t \omega}=D_{i t \omega}+I_{i t \omega}^{+}, \quad i \in I, t \in T, \omega \in W \\
L_{i t \omega} \leq D_{i t \omega}, \quad i \in I, t \in T, \omega \in W \\
\sum_{i \in I}\left(P_{i t} \cdot Q_{i t}+S_{i t \omega} \cdot Y_{i t}\right) \leq C_{t \omega}+O_{t \omega}, \quad t \in T, \omega \in W \\
O_{t \omega} \leq U_{t}, \quad t \in T, \omega \in W \\
I_{i t \omega}^{+}, L_{i t \omega}, O_{t \omega} \geq 0, \quad i \in I, t \in T, \omega \in W
\end{array}\right.
$$

A função objetivo (9) do modelo de programação estocástica minimiza o custo total esperado incorrido com as decisões de primeiro e segundo estágios. 0 primeiro termo da função objetivo refere-se ao custo de primeiro estágio, ao passo que o segundo termo representa o custo esperado ou de segundo estágio. As restrições (10) a (14) são similares às suas versões determinísticas, mas devem ser satisfeitas para todo cenário $\omega$ e $W$. 0 modelo (9) a (14) é denominado risco neutro (RN) para diferenciar do modelo com aversão ao risco a ser apresentado na seção 5 .

No modelo RN, a matriz tecnológica associada às variáveis de primeiro estágio varia com $\omega$, uma vez que o parâmetro $S_{\text {ito }}$ é estocástico. Além disso, a matriz tecnológica associada às variáveis de segundo estágio é aleatória devido ao parâmetro $R_{i t \text { to }}$; nesse caso, o modelo (9) a (14) é dito possuir recurso aleatório ou recurso geral (Sem \& Higle, 1999). Em relação à factibilidade, o modelo (9) a (14) é dito possuir recurso relativamente completo, i.e., o custo total (ou o valor de recurso) é finito para todo cenário $\omega \in W$, considerando apenas as variáveis de primeiro estágio satisfazendo as restrições (4) e (8).

\subsection{Valor esperado de informação perfeita e valor da solução estocástica}

No que diz respeito à análise do modelo estocástico, existem duas medidas conhecidas como valor esperado da informação perfeita (EVPI) e valor da solução estocástica (VSS). De acordo com Birge \& Louveaux (1997), o EVPI representa o valor máximo que o tomador de decisão estaria disposto a pagar a fim de descobrir o valor exato de uma variável aleatória - a informação perfeita - antes de tomar sua decisão. Já o VSS corresponde ao valor "economizado" (problemas de minimização de custos) ou ganho (problemas de maximização de lucro) por se utilizar o modelo estocástico ao invés de basear-se a produção de acordo com os valores esperados das variáveis aleatórias.

0 cálculo do EVPI é realizado da seguinte maneira: (i) Determine a solução ótima do modelo determinístico $Z_{\omega}$ para cada cenário $\omega$. lsso equivale a determinar os custos do planejamento da produção sob informação perfeita. (ii) Obtenha a expectativa dos custos sob informação perfeita $W S=\sum_{\omega \in W} \pi_{\omega} \cdot Z_{\omega}$. (iii) Calcule $\mathrm{EVPI}=\mathrm{RP}-\mathrm{WS}$, em que RP é o valor ótimo do problema de dois estágios com recurso.

Para se calcular o VSS: (i) Resolva o problema do valor esperado EV, i.e., o problema determinístico 1 a 8 cujos parâmetros estocásticos são substituídos pelos correspondentes valores esperados. (ii) Fixe as variáveis de primeiro estágio $Q_{i t}$ e $Y_{i t}$ do problema RP de acordo com os valores obtidos pelo problema EV. O valor ótimo desse problema em que o primeiro estágio foi previamente determinado é conhecido como EEV. (iii) Determine EEV. (iv) Calcule VSS = EEV - RP.

Embora o problema de programação estocástica possua recurso relativamente completo, o problema EEV pode não ter solução factível. De fato, a solução do problema EV não é, necessariamente, factível para o problema de programação estocástica de dois estágios quando o recurso é relativamente completo. Como consequência, o problema EEV pode ser infactível para algum cenário. Nesse caso, é usual assumir VSS $\rightarrow \infty$. Note que, se $U_{t}$ for suficientemente grande, então é possível garantir que o problema EEV será factível, independentemente da factibilidade das variáveis de decisão de primeiro estágio. Nesse caso, o problema de programação estocástica possui recurso completo.

0 leitor interessado em se aprofundar nesses conceitos pode consultar Kall \& Wallace (1994), Birge \& Louveaux (1997) e Escudero et al. (2007), entre outros. 


\section{Modelo de programação estocástica com aversão ao risco}

No modelo RN (9) a (14), a função objetivo é dita ser neutra em relação ao risco, pois apenas um valor esperado é minimizado. De acordo com Shapiro (2009), isso pode ser justificado quando vale a Lei dos Grandes Números (se a Lei dos Grandes Números é válida, então, após um número suficientemente grande de repetições de um experimento aleatório, a média dos resultados dos experimentos converge com probabilidade um para o seu valor esperado teórico) e o interesse é em um desempenho do modelo em longo prazo, sem se preocupar com as flutuações específicas de uma variável aleatória. Vladimirou \& Zenios (1997) preconizam que, na prática, pode ser muito custoso ou mesmo infactível reimplementar uma decisão a cada realização das variáveis aleatórias. Nesse caso, os autores defendem que é crucial reduzir a variabilidade das decisões de segundo estágio dentro de um programa estocástico com múltiplos cenários.

Neste trabalho é proposto um modelo do tipo média-risco para controlar a variabilidade dos custos de segundo estágio e isso é interessante do ponto de vista prático, pois esse tipo de modelo incorpora a noção de minimizar a variabilidade dos custos de segundo estágio em relação a algum cenário de referência, minimizando também os custos que possam incorrer caso o planejamento da produção necessite de reimplementação. A ideia básica é combinar a expectância e a dispersão da função objetivo:

$\min _{x} E[f(x, \omega)]+\varphi \cdot D[f(x, \omega)]$

em que a função objetivo $f(x, \omega)$ é caracterizada pela expectância $\mathrm{E}[\cdot]$ e pela dispersão estatística $\mathrm{D}[\cdot]$ (variância, desvio padrão etc.), e $\phi$ é um peso não negativo que reflete as preferências do decisor em relação ao risco. Nesse trabalho, utilizou-se a medida de variabilidade conhecida por Upper Partial Mean (UPM), ou média parcial superior (Ahmed \& Sahinidis, 1998; Miranda, 2007; Alem \& Morabito, 2012). Basicamente, essa medida de variabilidade penaliza somente os desvios positivos de cada cenário em relação ao custo esperado de segundo estágio, i.e., o cenário de referência é o próprio valor esperado do problema estocástico de dois estágios. A formulação matemática do problema de programação estocástica com aversão ao risco do tipo média-risco com medida de variabilidade (MR) pode ser escrita como:

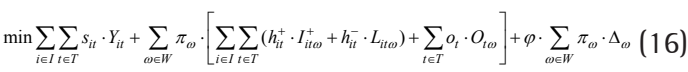

$$
\text { s.a.: }\left\{\begin{array}{l}
\text { Restrições determinísticas (4) e (8) } \\
\text { Restrições estocásticas }(10),(11),(12),(13) \mathrm{e}(14) \\
\Delta_{\omega} \geq \sum_{i \in I} \sum_{t \in T}\left(h_{i t}^{+} \cdot I_{i t \omega}^{+}+h_{i t}^{-} \cdot L_{i t \omega}\right)+\sum_{t \in T} o_{t} \cdot O_{t \omega}- \\
\quad-\sum_{\omega^{*} \in W} \pi_{\omega^{*}} \cdot\left[\sum_{i \in I} \sum_{t \in T}\left(h_{i t}^{+} \cdot I_{i t \omega^{*}}^{+}+h_{i t}^{-} \cdot L_{i t \omega^{*}}\right)+\sum_{t \in T} o_{t} \cdot O_{t \omega^{*}}\right], \omega \in W \\
\Delta_{\omega} \geq 0, \quad \omega \in W
\end{array}\right.
$$

A função objetivo do tipo média-risco (16) minimiza o custo total esperado (média) e o desvio esperado $\Sigma_{\omega} \pi_{\omega} \cdot \Delta_{\omega}$ (risco) ponderado pelo peso multicritério $\phi$. A restrição (17) define a medida de risco, i.e., a dispersão do custo total de segundo estágio de cada cenário em relação ao custo total esperado. Por fim, como esse é um problema de minimização de custos, a restrição (18) garante que desvios são não negativos. Note que os dois objetivos conflitantes na função objetivo, minimizar simultaneamente a média e o risco, definem um problema de otimização multicritério. Para $\phi=0$, a parcela referente ao risco é ignorada e os modelos RN (9) a (14) e MR (16) a (18) são equivalentes. Nesse caso, espera-se um custo total menor, mas com um desvio maior. À medida que $\phi$ é incrementado, o custo total aumenta, mas o desvio é reduzido. A princípio, não há um valor máximo para $\phi$. Diz-se, simplesmente, que quanto maior é esse valor, mais o decisor é averso ao risco, no sentido de que ele está mais disposto a sacrificar o custo total esperado em troca da obtenção de soluções com menor variabilidade. Em geral, são resolvidos vários modelos $\mathrm{MR}$, um para cada valor de $\phi$ num intervalo definido, para ser possivel determinar as curvas de tradeoff entre o custo total e o risco. Assim, o decisor pode fazer a escolha de $\phi$ de acordo com as suas preferências em relação ao risco.

\section{Experimentos computacionais}

Os experimentos computacionais executados neste trabalho objetivaram: ( $i$ ) avaliar o desempenho dos modelos determinístico, estocástico e com aversão ao risco; (ii) aferir o valor da solução estocástica e da informação perfeita; (iii) analisar as decisões de segundo estágio e os níveis de serviço em cada cenário; ( $i v)$ entender o tradeoff entre custo e risco. Valores de demandas, tempos de setup, tempos de produção e custos foram baseados em um exemplo real de uma unidade produtora de suplementos para animais com 26 famílias de produtos e uma carteira de pedidos constituída por nove períodos (meses) do horizonte de planejamento. Os tempos de setup foram determinados de acordo com a média dos tempos de setup dependentes da sequência. Com base nos dados históricos da planta de suplementos para nutrição 
animal e dados de perecibilidade encontrados na literatura (Costa et al., 2014), considerou-se uma taxa de perecibilidade de 5\% ao mês para a modelagem determinística. Os modelos matemáticos foram codificados no Sistema de Modelagem Algébrica GAMS e resolvidos pelo solver CPLEX $11.2 \mathrm{com}$ parâmetros default de resolução em um notebook Core i3, 4.0 GB de RAM, 2.0 GHz e plataforma Windows VISTA. Todas as instâncias foram resolvidas em menos de 15 minutos, permitindo-se um gap de otimalidade de $0,1 \%$.

\subsection{Representação dos cenários}

Para gerar a árvore de cenários ilustrada na Figura 2, considerou-se que as três variáveis aleatórias (demandas, tempos de setup e taxas de perecibilidade) podem assumir apenas três possíveis realizações ou valores. Cada valor foi definido qualitativamente por Alto (A), Médio (M) ou Baixo (B), tendo como referência o correspondente valor médio ou nominal. Considerando-se independência estatística entre as variáveis aleatórias, obteve-se uma árvore de cenários constituída por 27 diferentes cenários. Note-se, por exemplo, que o primeiro cenário da Figura 2 refere-se à demanda alta, perecibilidade alta e tempo de setup alto. As realizações associadas às demandas nos cenários A, M e B foram geradas a partir de uma distribuição uniforme nos intervalos $\left[1,05 . D_{i t} ; 1,3 . D_{i t}\right]$, $\left[0,95 . D_{i t} ; 1,05 . D_{i t}\right]$ e $\left[0,7 . D_{i t} ; 0,95 . D_{i t}\right]$, respectivamente. As realizações A, M e B associadas aos tempos de preparação foram geradas de maneira análoga, segundo uma distribuição uniforme nos intervalos $\left[1,05 . S_{i t} ; 1,2 . S_{i t}\right],\left[0,95 . S_{i t} ; 1,05 . S_{i t}\right]$ e $\left[0,8 . S_{i t} ; 0,95 . S_{i t}\right]$, respectivamente. Finalmente, a taxa de perecibilidade foi gerada uniformemente nos intervalos $\left[1,2 . R_{i t} ; 2 \cdot R_{i l}\right],\left[0,8 \cdot R_{i t} ; 1,2 \cdot R_{i t}\right] \mathrm{e}$ $\left[0,4 . R_{i t} ; 0,8 . R_{i t}\right]$. Note-se que $D_{i t}, S_{i t}$ e $R_{i t}$ são os correspondentes parâmetros determinísticos.

Devido à tendência modesta atual de crescimento do setor de nutrição animal (Sindicato Nacional da Indústria de Alimentação Animal, 2013), as probabilidades de ocorrência das realizações $A$, M e $B$ foram assumidas 0,3, 0,5 e 0,2, respectivamente. Por outro lado, devido às altas temperaturas brasileiras, à facilidade de ocorrência de contaminação dos produtos e processos e à dificuldade de reduzir-se a perecibilidade de itens orgânicos sem grandes investimentos, as probabilidades das realizações $A$, $\mathrm{M}$ e $\mathrm{B}$ da taxa de perecibilidade foram consideradas $0,5,0,3$ e 0,2 , respectivamente. Para os tempos de setup, as ocorrências A, M e B foram consideradas equiprováveis. Uma vez que as variáveis aleatórias são independentes, as probabilidades dos 27 cenários foram facilmente calculadas por meio da regra do produto. Dessa forma, a probabilidade de ocorrência do cenário 1 é igual ao produto das probabilidades dos três parâmetros estocásticos serem altos, i.e., $0,3 \cdot 0,5 \cdot(1 / 3)=0,05$.

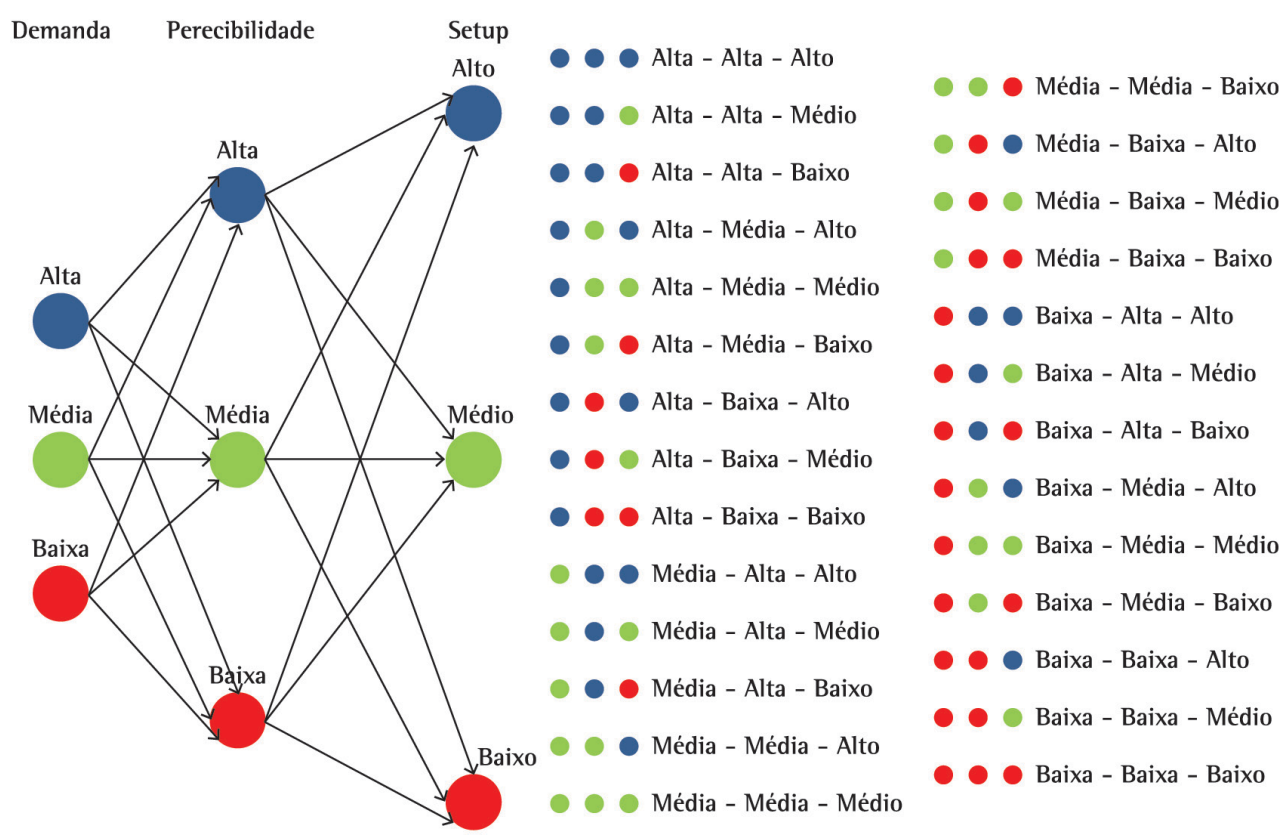

Figura 2. Árvore de cenários como combinação de três realizações (Alta, Média e Baixa) para cada um dos parâmetros estocásticos (demanda, taxa de perecibilidade e tempo de preparação). 


\subsection{Análise dos resultados do modelo determinístico}

Os resultados obtidos com o modelo determinístico representam adequadamente a dinâmica do planejamento agregado da produção na unidade de suplementos vitamínicos. Como os custos de lost sales são maiores do que os custos de estocagem e horas extras, o planejamento ótimo da produção busca atender a demanda do período atual e utilizar a capacidade restante para produzir produtos demandados em períodos futuros cuja capacidade de produção não se mostra suficiente para atender a demanda. No entanto, estocar significa perder parte dos lotes de produção devido à perecibilidade e, por essa razão, a solução ótima evita altos níveis de estoque. Em geral, as horas extras são utilizadas nos períodos em que a demanda é maior do que a capacidade disponível.

\subsection{Análise dos resultados do modelo de programação estocástica}

A Tabela 1 apresenta as probabilidades $\left(\pi_{\omega}\right)$, os custos de estoque (IC), os níveis dos estoques (1), os custos de lost sales (LSC), as lost sales (LS), os custos de horas extras (OC), as horas extras utilizadas (0) e o nível de serviço $\beta$ para os 27 cenários. 0 nível de serviço tipo 11 (taxa de atendimento da demanda) avalia a demanda total que não foi atendida ao final do último período em cada um dos cenários $\omega$ : $\beta_{\omega}=\left[1-L_{\omega}\right] / D_{\omega}$, sendo $L_{\omega}=\sum_{i \in I} L_{i T \omega}, L_{\text {iT } \omega}$ representa o lost sales do último período $T$ e $D_{\omega}=\sum_{i \in I} \sum_{t \in T} D_{i t \omega}$.

Na última linha da tabela são exibidos o custo total esperado, assim como o custo de preparação de primeiro estágio.

Nos cenários de demanda alta 1 a 9, o volume (e o custo total) de estoque é menor. Esse custo representa, aproximadamente, $30 \%$ do custo de estoque dos cenários com demanda média e apenas $2 \%$ do mesmo custo no caso de demanda baixa. Esse resultado era esperado, pois as demandas altas consomem praticamente toda a capacidade dos respectivos períodos correntes e há pouca margem de produção para estocar e suprir posteriores déficits. Por essa razão é razoável que os níveis de lost sales sejam maiores quando a demanda é alta e, assim, o nível de serviço desses cenários tem o pior desempenho (84\%, em média). Para os cenários com demanda média, o nível de serviço alcançou um desempenho

Tabela 1. Resultados do modelo estocástico neutro ao risco (9) a (14).

\begin{tabular}{|c|c|c|c|c|c|c|c|c|}
\hline Cenário & $\pi_{\omega}$ & IC & 1 & LSC & LS & $\mathrm{OC}$ & 0 & $\beta \%$ \\
\hline 1. Alta-Alto-Alta & 0,050 & 7.201 & 142 & 251.287 & 1416 & 13.675 & 159 & $84,9 \%$ \\
\hline 2. Alta-Alto-Média & 0,050 & 7.133 & 141 & 278.368 & 1548 & 13.490 & 157 & $83,7 \%$ \\
\hline 3. Alta-Alto-Baixa & 0,050 & 6.723 & 131 & 289.306 & 1594 & 13.306 & 154 & $83,3 \%$ \\
\hline 4. Alta-Médio-Alta & 0,030 & 7.179 & 143 & 281.205 & 1577 & 13.647 & 159 & $83,5 \%$ \\
\hline 5. Alta-Médio-Média & 0,030 & 7.191 & 141 & 255.250 & 1401 & 13.496 & 157 & $85,1 \%$ \\
\hline 6. Alta-Médio-Baixa & 0,030 & 7.057 & 138 & 277.117 & 1535 & 13.320 & 155 & $83,9 \%$ \\
\hline 7. Alta-Baixo-Alta & 0,020 & 6.474 & 127 & 279.893 & 1534 & 13.697 & 159 & $83,9 \%$ \\
\hline 8. Alta-Baixo-Média & 0,020 & 6.655 & 129 & 283.033 & 1552 & 13.486 & 157 & $83,7 \%$ \\
\hline 9. Alta-Baixo-Baixa & 0,020 & 6.741 & 130 & 280.580 & 1538 & 13.272 & 154 & $83,8 \%$ \\
\hline 10. Média-Alto-Alta & 0,083 & 23.203 & 357 & 19.212 & 115 & 13.664 & 159 & $98,6 \%$ \\
\hline 11. Média-Alto-Média & 0,083 & 22.679 & 410 & 12.936 & 53.7 & 13.521 & 157 & $99,3 \%$ \\
\hline 12. Média-Alto-Baixa & 0,083 & 17.218 & 321 & 23.804 & 140 & 13.311 & 155 & $98,3 \%$ \\
\hline 13. Média-Médio-Alta & 0,050 & 29.877 & 424 & 22.285 & 130 & 13.703 & 159 & $98,4 \%$ \\
\hline 14. Média-Médio-Média & 0,050 & 21.492 & 380 & 20.828 & 126 & 13.476 & 157 & $98,4 \%$ \\
\hline 15. Média-Médio-Baixa & 0,050 & 21.277 & 356 & 20.898 & 115 & 13.287 & 154 & $98,6 \%$ \\
\hline 16. Média-Baixo-Alta & 0,033 & 24.082 & 376 & 17.750 & 103 & 13.694 & 159 & $98,7 \%$ \\
\hline 17. Média-Baixo-Média & 0,033 & 23.206 & 391 & 26.596 & 142 & 13.486 & 157 & $98,2 \%$ \\
\hline 18. Média-Baixo-Baixa & 0,033 & 20.851 & 356 & 23.432 & 129 & 13.292 & 155 & $98,4 \%$ \\
\hline 19. Baixa-Alto-Alta & 0,033 & 357.959 & 5.835 & 30,2 & 0.16 & 13.632 & 159 & $100 \%$ \\
\hline 20. Baixa-Alto-Média & 0,033 & 321.212 & 5.307 & 9,3 & 0.1 & 13.512 & 157 & $100 \%$ \\
\hline 21. Baixa-Alto-Baixa & 0,033 & 332.255 & 5.462 & 121 & 0.37 & 13.268 & 154 & $100 \%$ \\
\hline 22. Baixa-Médio-Alta & 0,020 & 368.227 & 6.151 & 153 & 0.53 & 13.688 & 159 & $100 \%$ \\
\hline 23. Baixa-Médio-Média & 0,020 & 357.424 & 5.768 & 57,5 & 0.17 & 13.501 & 157 & $100 \%$ \\
\hline 24. Baixa-Médio-Baixa & 0,020 & 390.623 & 6.325 & 56,7 & 0.17 & 13.303 & 155 & $100 \%$ \\
\hline 25. Baixa-Baixo-Alta & 0,013 & 394.543 & 6.566 & 37,0 & 0.11 & 13.630 & 157 & $100 \%$ \\
\hline 26. Baixa-Baixo-Média & 0,013 & 394.867 & 6.438 & 39,1 & 0.11 & 13.479 & 157 & $100 \%$ \\
\hline 27. Ваiха-Baixo-Ваiха & 0,013 & 370.554 & 5.811 & 133 & 0.42 & 13.289 & 155 & $100 \%$ \\
\hline Média & - & 84.684 & 1.390 & 92.357 & 513 & 13.484 & 157 & $94,5 \%$ \\
\hline
\end{tabular}


médio de 98,5\%. Finalmente, nos cenários com demanda baixa, os níveis de serviço alcançaram $100 \%$ e o volume de estoque é bem maior do que nos cenários remanescentes. Esses resultados sugerem que o nível de atendimento da demanda depende da utilização dos estoques, principalmente no intuito de suprir demandas em períodos nos quais a capacidade produtiva é restrita.

Como a capacidade de produção representa 64 horas por mês, ao longo dos nove meses de horizonte de planejamento, o nível de utilização das horas extras para todos os cenários mostrou-se relativamente baixo (em média 28\% do total disponível). Como no modelo determinístico, existe uma tendência em se utilizar as horas extras somente quando a capacidade disponível não é suficiente para suprir a demanda, i.e., o modelo evita incorrer em horas extras para produzir excedente de produção de períodos futuros. Corroborando essa afirmação, nos períodos 2 , 5 e 9 , em que a demanda é proporcionalmente mais alta, a utilização de horas extras chegou a 99,9\% e, no restante dos períodos, não houve utilização delas. Além disso, os cenários com maior utilização de horas extras foram aqueles cujos tempos de setup são altos, seguidos pelos cenários cujos tempos de setup são médios e, por fim, pelos cenários cujos tempos de setup são baixos. A Tabela 1 mostra que essa utilização é repetida, independentemente da variação dos outros parâmetros e, a partir da análise dos resultados, percebeu-se que o incremento do uso de horas extras corresponde ao acréscimo de tempo na realização dos setups.

Por fim, como os níveis de serviço e estoque são extremamente dependentes das variações da demanda, do ponto de vista da indústria de nutrição animal é importante investir na criação de um histórico estruturado de pedidos colocados e dos efetivamente entregues. 0 investimento na busca de acurácia de previsão de demanda é o que trará maior impacto no aproveitamento dos recursos.

\subsection{Análise do EVPI e VSS}

A análise do EVPI mostrou que a economia proveniente da informação perfeita é bastante relevante, chegando a $82,3 \%$ do custo total esperado da solução estocástica. Na programação da produção, essa economia representa um aumento médio de $4 \%$ da necessidade de horas extras e, por outro lado, uma redução de $99 \%$ da quantidade de lost sales e de $73 \%$ da necessidade de estocagem no conjunto de cenários. Considerando-se que as lost sales oneram mais a função objetivo do problema, não é surpreendente que a taxa de redução dessa variável de segundo estágio seja mais elevada que das outras variáveis sob informação perfeita. Nos cenários em que a demanda é baixa, a informação perfeita provê soluções 95\% menores do que do modelo estocástico, em média. Já nos cenários com demanda média, essa proporção é de aproximadamente 86\% e, para os cenários com demandas altas, os custos sob informação perfeita são 68\% dos custos alcançados com o modelo estocástico.

No que diz respeito aos cenários em que os tempos de setup e as taxas de perecibilidade variam, o modelo mostrou que a informação perfeita traz, em média, aproximadamente $83 \%$ de economia em comparação com os resultados do modelo estocástico. A Figura 3 apresenta os custos totais médios alcançados sob informação perfeita para variações nos parâmetros incertos e mostra uma comparação com o custo do problema estocástico RP. Note-se que a variação da

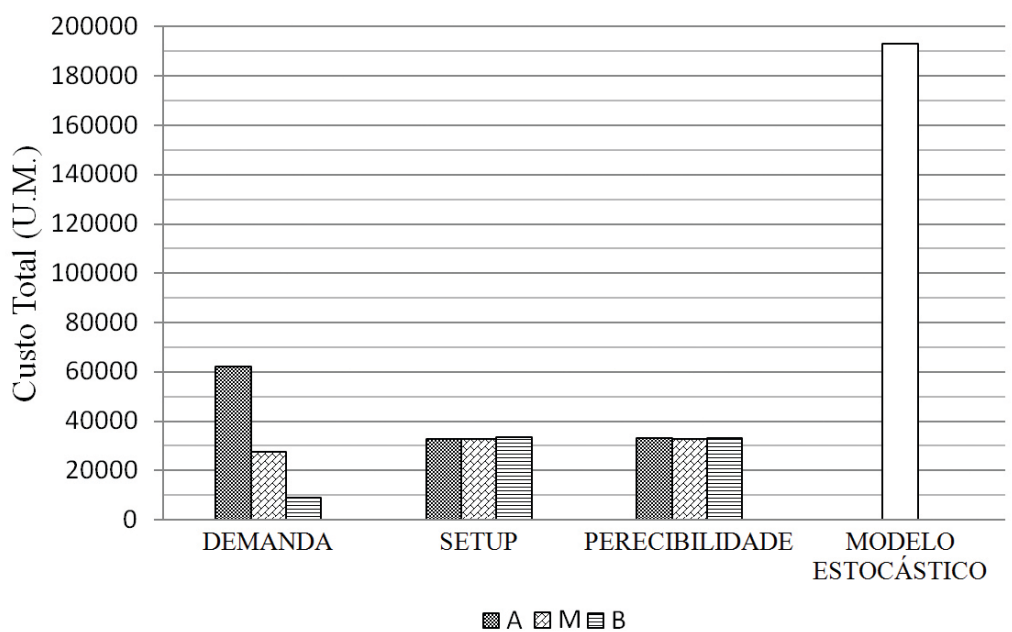

Figura 3. Comparação dos custos médios sob informação perfeita com o modelo RP. 
demanda (alta, média e baixa) tem maior impacto nos custos do que a variação dos tempos de setup e taxas de perecibilidade. Conforme esperado, os problemas determinísticos, wait-and-see, não são boas aproximações do problema estocástico RP, principalmente em cenários mais pessimistas, uma vez que nesses cenários a aleatoriedade tem maior impacto e o conhecimento prévio das variáveis aleatórias pode significar o sucesso do planejamento.

A análise do VSS evidenciou a precariedade das decisões com base nos valores médios do problema EV. Nesse contexto, é importante pontuar que em quase todos os cenários foi possível encontrar uma solução factível para o problema EEV sem ser necessário o aumento da capacidade de produção. Essa incapacidade de geração de um plano de produção ocorreu apenas no cenário em que a demanda, o tempo de setup e a perecibilidade são altos, o que sugere que a restrição de capacidade da unidade produtiva é a maior responsável pela inviabilidade de se encontrar um plano de produção. Em outras palavras, nos períodos de alta demanda, a demora na preparação do misturador, somada ao tempo gasto com o processamento dos produtos, excederia a capacidade total e as horas extras disponíveis. Nesse caso, diz-se que as decisões de valor médio do problema EV são

Tabela 2. Resultados do modelo de programação estocástica com aversão ao risco (16) a (18).

\begin{tabular}{|c|c|c|c|c|c|c|}
\hline$\phi$ & $\mathrm{FO}_{\phi}$ & Preço (\%) & $\bar{\Delta}_{\phi}$ & $\bar{\Delta}(\%)$ & $\mathrm{DP}_{\phi}$ & DP (\%) \\
\hline 0 & 193.283 & 0 & 67.318 & 0 & 139.071 & 0 \\
\hline 0,1 & 199.982 & 3,35 & 65.852 & 2,178 & 134.803 & 3,069 \\
\hline 0,2 & 206.481 & 6,39 & 65.546 & 2,633 & 134.475 & 3.304 \\
\hline 0,3 & 213.035 & 9,27 & 64.769 & 3,786 & 133.583 & 3,946 \\
\hline 0,4 & 219.407 & 11,91 & 62.882 & 6,589 & 129.774 & 6,685 \\
\hline 0,5 & 225.394 & 14,25 & 58.696 & 12,81 & 118.487 & 14,80 \\
\hline 0,6 & 231.192 & 16,40 & 57.361 & 14,79 & 114.550 & 17,63 \\
\hline 0,7 & 236.770 & 18,37 & 55.847 & 17,04 & 111.698 & 19,68 \\
\hline 0,8 & 242.279 & 20,22 & 53.346 & 20,75 & 106.715 & 23,27 \\
\hline 0,9 & 247.580 & 21,93 & 52.833 & 21,52 & 105.460 & 24,17 \\
\hline 1 & 252.897 & 23,57 & 50.975 & 24,28 & 100.395 & 27,81 \\
\hline 1,1 & 257.842 & 25,04 & 49.763 & 26,08 & 97.700 & 29.75 \\
\hline 1,2 & 262.804 & 26,45 & 49.089 & 27,08 & 96.233 & 30,80 \\
\hline 1,3 & 267.630 & 27,78 & 45.272 & 32,75 & 89.622 & 35,56 \\
\hline 1,4 & 271.901 & 28,91 & 43.761 & 34,99 & 86.645 & 37,70 \\
\hline 1,5 & 276.078 & 29,99 & 40.436 & 39,93 & 78.993 & 43,20 \\
\hline 1,6 & 279.990 & 30,97 & 34.698 & 48,46 & 68.680 & 50,61 \\
\hline 1,7 & 283.264 & 31,77 & 30.456 & 54,76 & 60.820 & 56,27 \\
\hline 1,8 & 286.062 & 32,43 & 28.088 & 58,28 & 57.525 & 58,64 \\
\hline 1,9 & 288.883 & 33,09 & 26.813 & 60,17 & 55.399 & 60,16 \\
\hline 2 & 291.447 & 33,68 & 25.429 & 62,22 & 52.516 & 62,24 \\
\hline 2,1 & 293.719 & 34,19 & 12.969 & 80,73 & 31.189 & 77,57 \\
\hline 2,2 & 294.757 & 34,43 & 10.267 & 84,75 & 28.392 & 79,58 \\
\hline 2,3 & 295.728 & 34,64 & 9.840 & 85,38 & 27.610 & 80,15 \\
\hline 2,4 & 296.615 & 34,84 & 9.140 & 86,42 & 23.171 & 83,34 \\
\hline 2,5 & 297.579 & 35,05 & 8.440 & 87,46 & 25.494 & 81,67 \\
\hline 2,6 & 298.335 & 35,21 & 7.542 & 88,80 & 18.231 & 86,89 \\
\hline 2,7 & 299.059 & 35,37 & 6.261 & 90,70 & 19.094 & 86,27 \\
\hline 2,8 & 299.626 & 35,49 & 5.673 & 91,57 & 16.109 & 88,42 \\
\hline 2,9 & 300.132 & 35,60 & 2.682 & 96,02 & 11.439 & 91,77 \\
\hline 3 & 300.415 & 35,66 & 2.596 & 96,14 & 11.084 & 92,03 \\
\hline 3,1 & 300.540 & 35,69 & 2.245 & 96,66 & 9.282 & 93,33 \\
\hline 3,2 & 300.824 & 35,75 & 2.132 & 96,83 & 12.493 & 91,02 \\
\hline 3,3 & 301.065 & 35,80 & 1.747 & 97,41 & 8.817 & 93,66 \\
\hline 3,4 & 301.204 & 35,83 & 1.345 & 98,00 & 7.224 & 94,81 \\
\hline 3,5 & 301.308 & 35,85 & 1.258 & 98,13 & 5.099 & 96,33 \\
\hline 3,6 & 301.398 & 35,87 & 916,1 & 98,64 & 6.693 & 95,19 \\
\hline 3,7 & 301.494 & 35,89 & 796,5 & 98,82 & 6.617 & 95,24 \\
\hline 3,8 & 301.523 & 35,90 & 729,4 & 98,92 & 4.008 & 97,12 \\
\hline 3,9 & 301.642 & 35,92 & 666,6 & 99,01 & 4.172 & 97,00 \\
\hline 4 & 301.703 & 35,94 & 558,7 & 99,17 & 3.824 & 97,25 \\
\hline
\end{tabular}


operacionalmente infactíveis para uma situação de múltiplos cenários e, matematicamente, representa-se essa situação por VSS $\rightarrow \infty$.

Para efeito de análise, foi incluída capacidade extra de produção no modelo por meio de uma variável artificial, da mesma forma que as horas extras de trabalho por período, o que permitiu o levantamento de um plano de produção para o cenário em questão. Ao se confrontar os dados de demanda com a utilização das horas extras adicionais, confirmou-se que a necessidade de utilização da variável artificial ocorreu nos meses 2 e 9 do horizonte de planejamento, justamente quando a demanda se encontra em seus maiores níveis. Os resultados também indicaram que os custos totais do planejamento da produção são mais sensíveis às variações da demanda. 0 incremento no custo quando a demanda varia de média para alta é de 34.496 u.m., e quando passa de baixa para média, é de 18.731 u.m. Analogamente, a perecibilidade incrementa o custo total de 561 u.m. quando ela passa de média para alta e, no entanto, quando passa de baixa para média o custo total diminui 562 u.m. 0 tempo de setup onera sutilmente a função objetivo, aumentando 233 u.m. quando a demanda varia de média para baixa e diminuindo 929 u.m. quando passa de baixa para média.

Analisando-se os valores, conclui-se que, se o gestor quer investir para adquirir informações mais confiáveis sobre as variantes do sistema, informações de perecibilidade e tempos de setup seriam um investimento mais conservador, visto que em qualquer cenário o retorno sobre o investimento seria em torno de 160.335 u.m. Por outro lado, um gestor mais arrojado poderia investir nas informações de demanda, apostando de antemão que a demanda seria média ou baixa, o que traria um retorno de 165.635 u.m. a 193.191 u.m.

\subsection{Análise dos resultados do modelo de programação estocástica com aversão ao risco}

Para avaliar o tradeoff entre a redução da variabilidade dos custos de segundo estágio e o acréscimo no custo total da função objetivo, o modelo 16 a 18 foi resolvido para níveis de aversão ao risco $\phi$ variando de 0 a 4 , com passos de 0,1 . A Tabela 2 apresenta os valores de $\phi$; o custo total esperado de acordo com o parâmetro de risco $\mathrm{FO}_{\phi}$; a variação percentual entre o custo total esperado do modelo estocástico RN e o modelo com aversão ao risco MR, calculada como: preço $(\%)=\left(\mathrm{FO}_{\phi}-\mathrm{FO}_{0}\right) \cdot 100 \% / \mathrm{FO}_{0}$, em que $\mathrm{FO}_{0}$ é o custo total esperado do modelo RN; o valor esperado $\bar{\Delta}_{\phi}=\Sigma_{\omega} \pi_{\omega} . \Delta_{\omega}$; a redução do desvio $\bar{\Delta}(\%)=\left(\bar{\Delta}_{0}-\bar{\Delta}_{\phi}\right) \cdot 100 \% / \bar{\Delta}_{0}$, em que $\bar{\Delta}_{0}$ é o desvio esperado do modelo RN; o desvio padrão $\mathrm{DP}_{\phi}$ dos custos totais nos diferentes cenários para cada nível de aversão ao risco, em que $\mathrm{DP}_{0}$ é o desvio

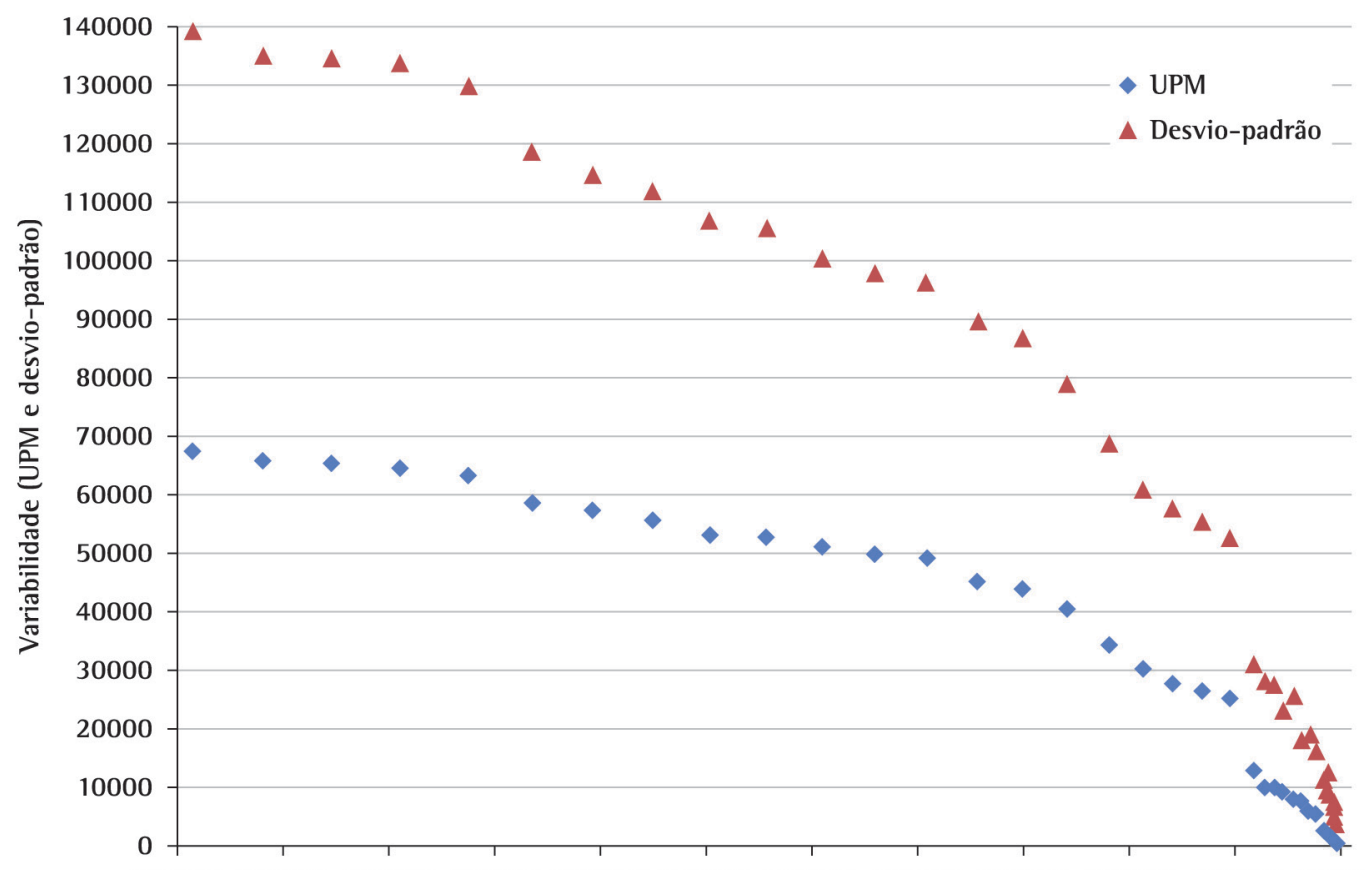

192000202000212000222000232000242000252000262000272000282000292000302000

Custo total esperado

Figura 4. Curvas de tradeoff entre o custo total e as medidas de variabilidade UPM e desvio padrão. 
padrão do modelo RN; a redução do desvio padrão $\mathrm{DP}(\%)=\left(\mathrm{DP}_{0}-\mathrm{DP}_{\phi}\right) .100 \% / \mathrm{DP}_{0}$. A Figura 4 ilustra as curvas de Pareto geradas para analisar o tradeoff entre o custo total esperado e as medidas de variabilidade UPM e desvio padrão. As Figuras 5, 6 e 7 exibem o comportamento dos níveis de estoque, utilização de horas extras e níveis de serviço, de acordo com o parâmetro de risco.

Como esperado, o custo total aumentou a cada incremento do nível de aversão ao risco. As variáveis de primeiro estágio não se alteraram com o aumento progressivo de $\phi$. No entanto, o valor de $\Delta$ sofreu decrementos sucessivos devidos às variações das variáveis de segundo estágio. Em geral, não é necessário ser muito conservador para alcançar soluç̧ões aversas ao risco, pois é possível garantir uma redução da dispersão dos custos de segundo estágio em mais de 50\%, comprometendo apenas 30\% do custo total esperado.

Note-se que os preços de aversão ao risco para $\phi$ de 0 até 0,7 são maiores do que as reduções dos custos de segundo estágio, i.e., o custo incremental incorrido na função objetivo é proporcionalmente maior que as reduções de variabilidade alcançadas. Quando $\phi$ assume valores iguais ou superiores a 0,8 , o preço percentual de aversão ao risco torna-se inferior ao incremento no custo total esperado, o que representa uma situação favorável para o decisor mais averso ao risco. Nesses casos, embora o custo total seja deteriorado 32,57\%, em média, é possível reduzir a variabilidade dos custos de segundo estágio em $72,15 \%$ e reduzir o desvio padrão dos custos totais esperados 70,87\%, em média. No planejamento da produção, tais reduções da variabilidade trazem maior segurança ao decisor no que diz respeito ao nível de força de trabalho a ser mantido pela empresa, demissões e contratações, e aos períodos em que serão utilizadas as horas extras.

Os resultados também indicaram que as soluções para $\phi=2,5$ e $\phi=3,2$ são dominadas pelas soluções obtidas com $\phi=2,4$ e $\phi=3,1$, respectivamente, uma vez que nesses dois casos o aumento da aversão ao risco ocasionou o aumento do desvio padrão e um custo total mais deteriorado. Uma explicação possivel para esse fenômeno é o fato de essas soluções apresentarem um gap de otimalidade de 0,1\%. Embora as soluções para $\phi=0,1, \phi=0,2$ e $\phi=0,3$ tenham aproximadamente a mesma variabilidade, os custos totais são mais deteriorados para as soluções à direita de $\phi=0,1$. Isso sugere que não vale a pena tomar decisões com base nas soluções fornecidas por $\phi=0,2$ e $\phi=0,3$.

Nas situações em que a proporção de aumento do custo total é maior do que a proporção de redução da variabilidade dos custos de segundo estágio $(0 \leq \phi \leq 0,7)$ houve um aumento de lost sales de 81 bateladas (de 514 para 595), o que representou uma queda do nível de serviço de 1\%. Em contrapartida, os volumes de estoque foram reduzidos em 207 bateladas (de 1.395 para 1.188). Mesmo com as economias

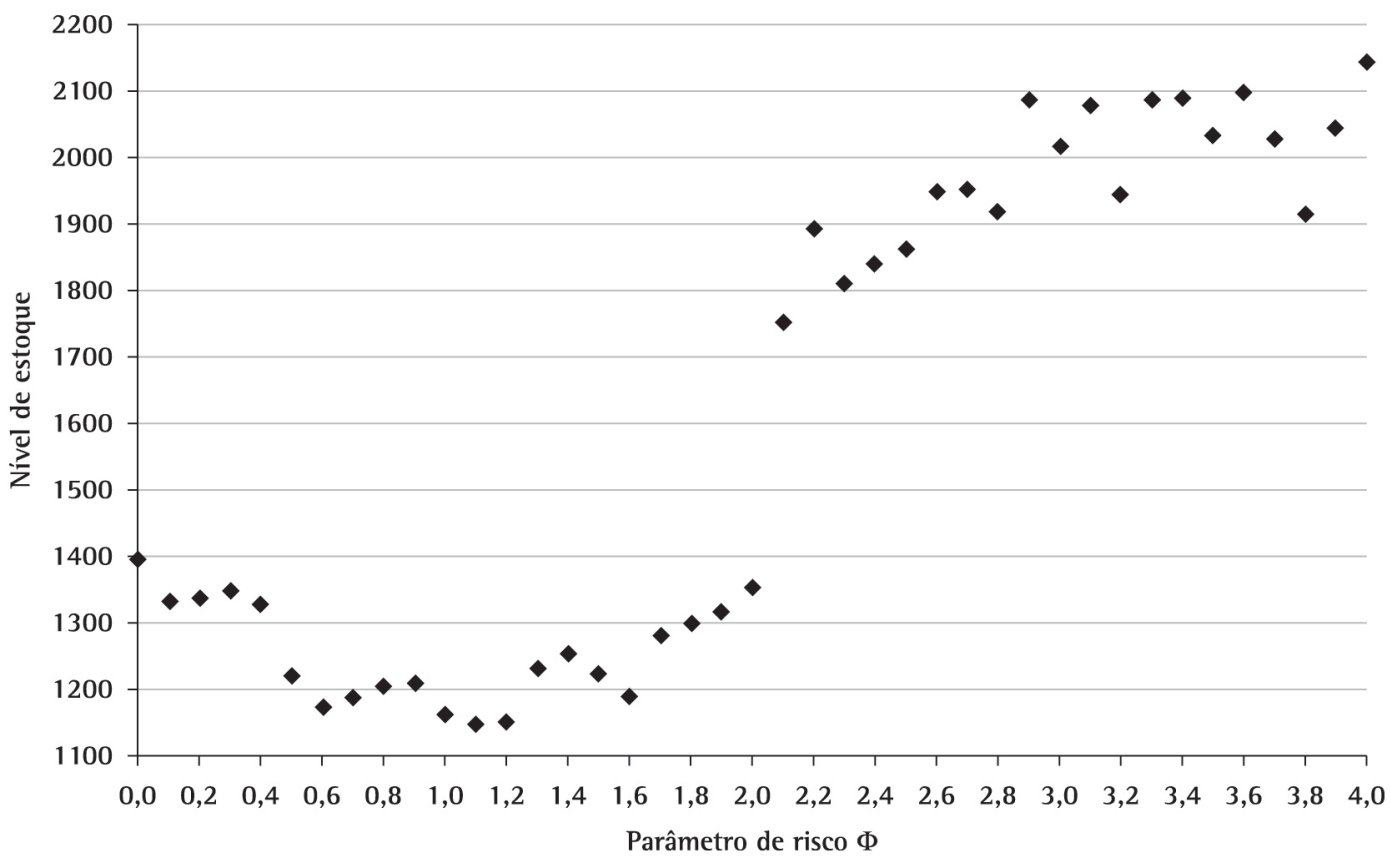

Figura 5. Nível de estoque fornecido pelo modelo de programação estocástica com aversão ao risco de acordo com o parâmetro de risco $\phi$. 
provenientes dessa redução de estoques, os custos totais aumentaram mais de $18,37 \%$ devido ao custo de lost sales. No intervalo em que a proporção de aumento do custo total é menor que a proporção de redução da variabilidade dos custos de segundo estágio
$(0,8 \leq \phi \leq 0,4)$, o volume de estoque aumentou cerca de $25 \%$ (de 1.281 para 1.699) e, simultaneamente, o nível de serviço reduziu-se em 2,9\%, por conta de 237 bateladas que não foram produzidas.

0 nível de horas extras apresentou três fases

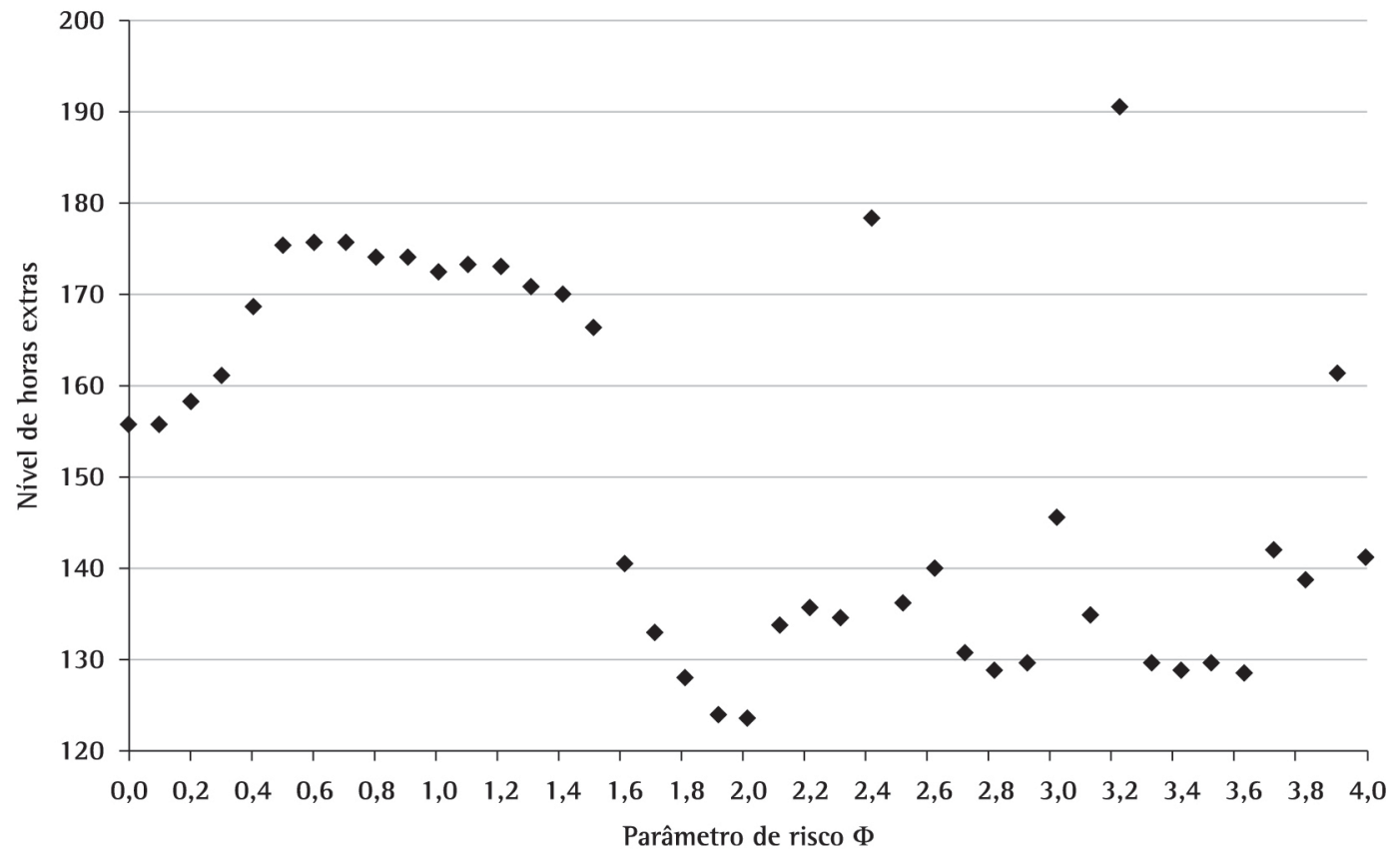

Figura 6. Nível de utilização de horas extras fornecido pelo modelo de programação estocástica com aversão ao risco de acordo com o parâmetro de risco $\phi$.

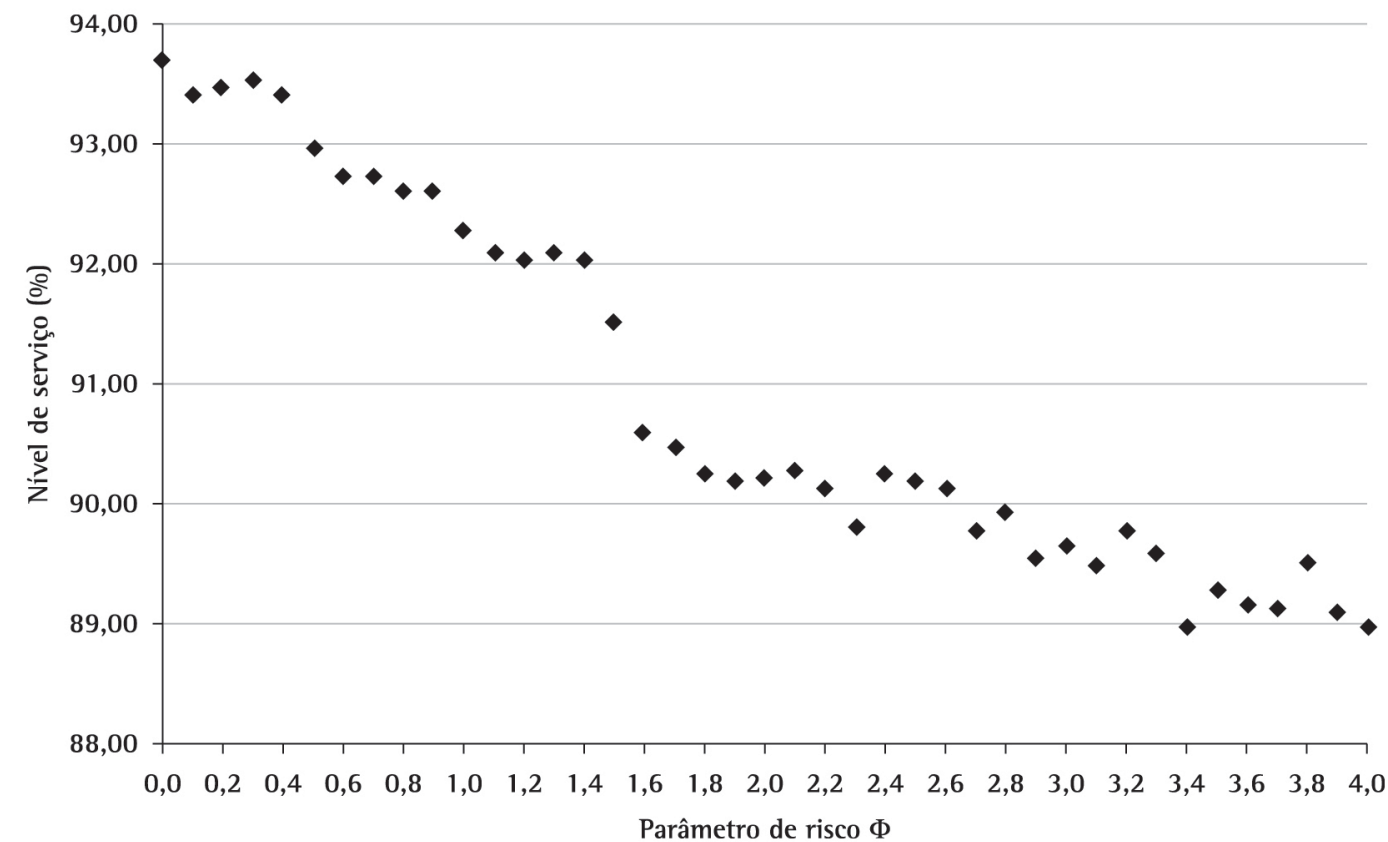

Figura 7. Nível de serviço (atendimento da demanda) fornecido pelo modelo de programação estocástica com aversão ao risco de acordo com o parâmetro de risco $\phi$. 
distintas. No intervalo $0 \leq \phi \leq 0,7$ houve uma tendência de aumento, passando de 155,7 na situação de neutralidade em relação ao risco para 175,6 horas em $\phi=0,7$. De $\phi=0,8$ até 2 , o nível de utilização de horas extras reduziu-se em, aproximadamente, $30 \%$, chegando a 123 horas. Na terceira fase, $2,1 \leq \phi \leq 4$, observa-se um comportamento relativamente estável em torno de 140 horas, com picos de utilização de horas extras de 178,6 horas em $\phi=2,4$ e 190,7 horas em $\phi=3,2$.

A Figura 8 ilustra a dispersão dos custos de segundo estágio obtidos a partir de um conjunto de 350 árvores de cenários geradas aleatoriamente, de acordo com os intervalos descritos na seção 6.1. Os correspondentes 350 exemplares foram resolvidos para diversos níveis de aversão ao risco e os 9.450 cenários (350×27 = 9450) foram analisados a partir da construção dos histogramas de frequência relativa. É interessante notar que a introdução da medida de variabilidade UPM reduz a probabilidade de se gerar um plano agregado de produção com custos muito elevados. Por exemplo, para $\phi=0$ há uma probabilidade não desprezível do custo total ser maior do que 400.000 u.m. Entretanto, tal probabilidade torna-se zero a partir de $\phi=1$. Por outro lado, a probabilidade de gerar planos de produção com custos baixos (e.g., menores do que 100.000 u.m.) também é reduzida, pois a medida de variabilidade proposta neste trabalho faz com que ambos os cenários mais otimistas e pessimistas aproximem-se do cenário de valor esperado.

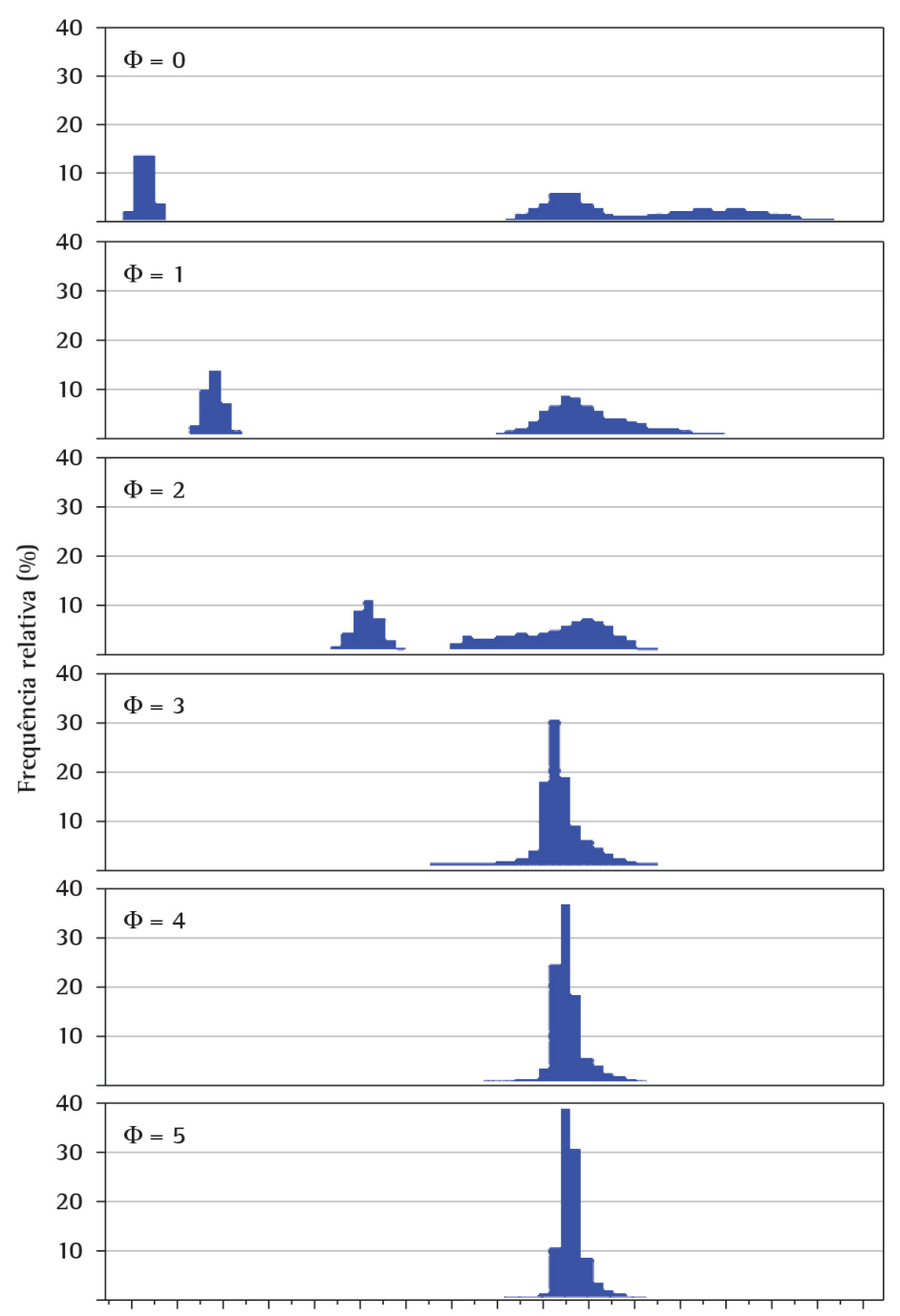

5075100125150175200225250275300325350375400425450

Custo total dos cenários (x 1000)

Figura 8. Histogramas do custo total do modelo com aversão ao risco sobre uma amostra de 9.450 cenários, considerando $\phi$ de 0 até 5 . 


\section{Considerações finais}

Neste trabalho foram propostos e analisados três modelos matemáticos para o problema de planejamento agregado da produção na indústria de nutrição animal. Além do modelo determinístico de dimensionamento de lotes com perecibilidade e lost sales, desenvolveu-se um modelo de programação estocástica de dois estágios com recurso para incorporar a aleatoriedade das demandas, tempos de preparação e taxas de perecibilidade, que são tipicamente dados difíceis de serem estimados com precisão. Por simplicidade, foram geradas três realizações discretas para cada parâmetro estocástico, as quais foram posteriormente combinadas numa estrutura em árvore para evidenciar possiveis correlações entre as variáveis aleatórias. Para gerar soluções menos sensiveis às realizações das variáveis aleatórias, apresentou-se um modelo estocástico com aversão ao risco cujo objetivo é controlar a redução da variabilidade dos custos de segundo estágio de acordo com a atitude dos decisores em relação ao risco.

De acordo com os experimentos numéricos executados com exemplares reais de uma planta de suplementos vitamínicos, inferiu-se que: (l) A utilização de horas extras é importante para suprir demandas de períodos correntes. (ii) É bastante custoso adquirir informação perfeita sobre as variáveis aleatórias antes de tomar uma decisão, especialmente sobre a demanda, uma vez que o EVPI vale aproximadamente $82 \%$ do problema RP. Isso mostra que a aleatoriedade dos dados tem um impacto relevante no problema em questão, refletindo também a praticidade da solução estocástica em detrimento das soluções wait-and-see. (iii) Aproximações baseadas em valores médios resultam em soluções operacionalmente infactíveis para o problema de programação estocástica, especialmente no cenário com tempos de preparação, demandas e perecibilidade altos. (iv) 0 custo total esperado é mais sensivel às variações das demandas em relação às variações dos tempos de setup e da perecibilidade. ( v) 0 "preço da aversão ao risco" mostrou-se razoável para soluções mais aversas ao risco. É possível, por exemplo, reduzir o UPM e o desvio padrão em mais de 90\%, deteriorando o custo total por volta de 35\%.

Pesquisas futuras incluem a proposição de métodos mais sofisticados para representar as incertezas e gerar os cenários, e.g., séries temporais, amostragem e moment matching (Di Domenica et al., 2009), a modelagem estocástica e com aversão ao risco de problemas integrados de planejamento e sequenciamento da produção na indústria de nutrição animal, considerando a perecibilidade como parâmetro estocástico, e outras medidas de risco, como o conditional value-at-risk (CVaR) e restrições baseadas em dominância estocástica de segunda ordem.

\section{Referências}

Ahmed, S., \& Sahinidis, N. V. (1998). Robust process planning under uncertainty. Industrial \& Engineering Chemistry Research, 37(5), 1883-1892. http://dx.doi.org/10.1021/ ie970694t

Ahumada, 0., \& Villalobos, J. R. (2008). Application of planning models in the agri-food supply chain: A review. European Journal of Operational Research, 196(1), 1-20. http://dx.doi.org/10.1016/j.ejor.2008.02.014

Alfares, H. K., Khursheed, S. N., \&t Noman, S. M. (2005). Integrating quality and maintenance decisions in a production-inventory model for deteriorating items. International Journal of Production Research, 43(5), 899911. http://dx.doi.org/10.1080/0020754042000298511

Amorim, P., Meyr, H., Almeder, C., \& Almada-Lobo, B. (2013). Managing perishability in production-distribution planning: a discussion and review. Flexible Services and Manufacturing Journal, 25(3), 389-413. http://dx.doi. org/10.1007/s10696-011-9122-3

Alem, D., \& Morabiro, R. (2013). Risk-averse twostage stochastic programs in furniture plants. $O R$ Spectrum, 35(4), 773-806 http://dx.doi.org/10.1007/ s00291-012-0312-5

Bellman, R. (1957). Dynamic programming. Princeton: Princeton University Press.

Bellman, R. E., \& Zadeh, L. A. (1970). Decision making in fuzzy environment. Management Science, 17(4), 141154. http://dx.doi.org/10.1287/mnsc.17.4.B141

Birge, J. R., \& Louveax, F. (1997). Introduction to stochastic programming. New York: Springer.

Brahimi, N., Dauzere-Peres, S., Najid, N. M., \& Nordli, A. (2006). Single item lot sizing problems. European Journal of Operational Research, 168(1), 1-16. http:// dx.doi.org/10.1016/j.ejor.2004.01.054

Charnes, A., \& Cooper, W. (1959). Chance constrained programming. Management Science, 6(1), 73-79. http:// dx.doi.org/10.1287/mnsc.6.1.73

Clark, A. R., Morabito, R., \& Toso, E. A. V. (2010). Production setup-sequencing and lot-sizing at an animal nutrition plant through ATSP subtour elimination and patching. Journal of Scheduling, 13(2), 111-121. http://dx.doi. org/10.1007/s10951-009-0135-7

Costa, A. M., Santos, L. M. R., Alem, D. J., \& Santos, R. H. S. (2014). Sustainable vegetable crop supply problem with perishable stocks. Annals of Operations Research, 219(1), 265-283.

Dantzig, G. (1955). Linear programming under uncertainty. Management Science, 1(3-4), 197-206. http://dx.doi. org/10.1287/mnsc.1.3-4.197

Di Domenica, N., Lucas, C., Mitra, G., \& Valente, P. (2009). Scenario generation for stochastic programming and simulation: a modelling perspective. IMA Journal of Management Mathematics, 20(1), 1-38. http://dx.doi. org/10.1093/imaman/dpm027

Drexl, A., \& Kimms, A. (1997). Lot sizing and scheduling: survey and extensions. European Journal of Operational Research, 99(2), 221-235. http://dx.doi.org/10.1016/ S0377-2217(97)00030-1

Escudero, L. F., Garin, A., Merino, M., \& Perez, G. (2007). The value of the stochastic solution in multistage problems. TOP, 15(1), 48-64. http://dx.doi.org/10.1007/s11750007-0005-4

Jans, R., \& Degraeve, Z. (2008). Modeling industrial lot sizing problems: a review. International Journal of Production Research, 46(6), 1619-1643. http://dx.doi. org/10.1080/00207540600902262 
Kall, P., \& Wallace, S. (1994). Stochastic programming. New York: Wiley.

Karimi, B., Ghomi, S. F., \& Wilson, J. (2003). The capacitated lot sizing problem: a review of models and algorithms. Omega, 31(14), 365-378. http://dx.doi.org/10.1016/ S0305-0483(03)00059-8

Miranda, J. L. (2007). Optimização em sistemas de processos químicos: generalização de modelos com planeamento e sequenciamento (Tese de doutorado). Lisboa: Universidade Técnica de Lisboa.

Mula, J., Poler, R., Garcia-Sabater, J. P., \& Lario, F. C. (2006). Models for production planning under uncertainty: A review. International Journal of Production Economics, 103(1), 271-285. http://dx.doi.org/10.1016/j. ijpe.2005.09.001

Mulvey, J., Vanderbei, R., \& Zenios, S. (1995). Robust optimization of large-scale systems. Operations Research, 43(2), 264-281. http://dx.doi.org/10.1287/ opre.43.2.264

Pahl, J., Vo $\beta$, S., \& Woodruff, D. (2011). Discrete lot-sizing and scheduling with sequence-dependent setup times and costs including deterioration and perishability constraints. In 44th Hawaii International Conference on System Sciences, Kauai, Hawaii.

Pochet, Y., \& Wolsey, L. (2006). Production planning by mixed integer programing. New York: Springer.

Sahinidis, N. V. (2004). Optimization under uncertainty: state-of-the-art and opportunities. Computers and Chemical Engineering, 28(6-7), 971-983. http://dx.doi. org/10.1016/j.compchemeng.2003.09.017

Sen, S., \& Higle, J. L. (1999). An introductory tutorial on stochastic linear programming models. Interfaces, 29(2), 33-61. http://dx.doi.org/10.1287/ inte.29.2.33
Shapiro, A., Dentcheva, D., \& Ruszczynski, A. (2009). Lectures on stochastic programming: modeling and theory. Philadelphia: SIAM. http://dx.doi. org/10.1137/1.9780898718751

Sindicato Nacional da Indústria de Alimentação Animal. (2013). Boletim Informativo do Setor. São Paulo: Sindiracões. Recuperado em 22 de junho de 2013, de http://sindiracoes.org.br

Toso, E. A. V., Morabito, R., \& Clark, A. R. (2008). Combinação de abordagens GLSP e ATSP para o problema de dimensionamento e sequenciamento de lotes de produção de suplementos para nutrição animal. Pesquisa Operacional, 28(3), 423-450. http://dx.doi.org/10.1590/ S0101-74382008000300003

Toso, E. A. V., Morabito, R., \& Clark, A. R. (2009). Lot-Sizing and sequencing optimisation at an animal-feed plant. Computers \& Industrial Engineering, 57(3), 813-821. http://dx.doi.org/10.1016/j.cie.2009.02.011

Verderame P. M., Elia, J. A., Li, J., \& Floudas, C. (2010). Planning and scheduling under uncertainty: a review across multiple sectors. Industrial \& Engineering Chemistry Research, 49(9), 3993-4017. http://dx.doi. org/10.1021/ie902009k

Vladimirou, H., \& Zenios, S. (1997). Stochastic linear programs with restricted recourse. European Journal of Operational Research, 101(1), 177-192. http://dx.doi. org/10.1016/0377-2217(95)00370-3

\section{Agradecimentos}

Os autores gostariam de agradecer aos dois revisores anônimos pelos comentários e sugestões que ajudaram a tornar o texto mais claro. Essa pesquisa contou com o apoio financeiro da Fundação de Amparo à Pesquisa do Estado de São Paulo (Fapesp, processo 2010/10133-0).

\title{
Production planning in the animal nutrition industry under uncertainty
}

\begin{abstract}
One of the greatest challenges of production planning in the animal nutrition industry is determining the amount of each product that should be produced during each period, given the perishability of the products, the manual execution of the setups and the need to adjust the production capacity in a stochastic demand environment that is characterized by the seasonality of the products and raw materials. This paper investigates an aggregate production planning problem in a plant that produces supplements for horses, cattle, pigs and poultry. To address this problem, we proposed an extension of the classical capacitated lot-sizing problem to incorporate decisions about lost sales and inherent uncertainties in production planning, such as demands, setup times and perishability. To generate solutions that are less sensitive to changes in scenarios, we also developed a risk-averse stochastic model with an absolute semi-deviation-based risk measure. An analysis of the expected value of perfect information and the value of the stochastic solution confirmed that the stochastic approach outperformed the deterministic approximations in handling uncertainty. Furthermore, the results indicated that it is possible to significantly reduce the variability of the second-stage costs without sacrificing the expected total cost.
\end{abstract}

\section{Keywords}

Capacitated Lot-sizing Problem with Lost Sales and Perishability. Two-stage Stochastic Programming. Risk Management. Animal Nutrition Industry. 